The ERS designates this

educational activity for a

maximum of 1 CME credit. For

information on how to earn

CME credits, see www.

ers-education.org/pages/

default.aspx?id=2161

\section{T. Berghmans}

Department of Intensive Care and Thoracic Oncology, Institut Jules Bordet, Université Libre de Bruxelles, Brussels, Belgium

Correspondence

Dr Thiern Berghmans, Institut Jules Bordet RueHéger-Bordet, 1, Bruxelles 1000 Belgium

thierry.berghmans@bordet.be

Competing interests None declared.

Malignant pleural mesothelioma (MPM) is a relatively rare neoplasm, the incidence of which is now growing due to the large use of asbestos in the last decades. Although asbestos is banned from most of the industrialised countries, it is still largely used in emerging ones suggesting the persistence of the MPM epidemic. Despite observed advances, MPM is still a difficult disease to be diagnosed and treated. Few active therapeutics are currently available and the role of surgery remains debatable. Outside of clinical trials, there is no definite place for radiotherapy. The combination of cisplatin and pemetrexed is nowadays the standard first-line chemotherapy regimen for good performance status patients. New promising therapeutic approaches are under investigation such as histone deacetylase inhibitors vorinostat and valproate. Guidelines recently developed by a combined European Respiratory Society and European Society of Thoracic Surgery Task Force are now available and can serve the pneumologists and oncologists in their daily practice.

\section{Introduction}

Development of malignant pleural mesothelioma (MPM) is related to asbestos exposure even if some patients have no such contact history, the proportion of asbestos-associated mesotheliomas varies in the literature from 16 to $90 \%$ [1]. This relationship has been known for more than $40 \mathrm{yrs}$ [2]. It is a relatively rare neoplasm but its incidence is expected to dramatically increase in the near future due to the widespread use of asbestos. The mean time interval from asbestos exposure to first symptoms can be as long as 40 yrs [3], justifying an expected mesothelioma peak between 2015 and 2020 in Europe, according to the country [4-6] while this peak has yet be reached in other countries such as the USA [7]. The number of expected deaths is considerable: 1,950-2,450 deaths. $\mathrm{yr}^{-1}$ between 2011 and 2015 in the UK, 71,000 deaths for the first half of the 21 st century in the USA. It is only during the last decade that the health authorities and decision makers have correctly assessed the importance and the impact on the health general status of this rare neoplasm that has a poor prognosis in most of the cases. Currently, asbestos is prohibited in the European Community but some countries are still large producers, with the top five including Russia, China, Kazakhstan, Brazil and Canada. Asbestos is used in these countries as well as in emerging countries, such as India and lessdeveloped nations, suggesting a persistence of the epidemic in the future.
HERMES syllabus link: module B.2.3 


\section{Aetiology}

Asbestos exposure is the main risk factor for developing MPM. The first studies showing an increased incidence of MPM in exposed patients were performed at the beginning of the 1960s [2]. The relative risk of exposed people is estimated to range between 4.0 and 23.7 for household exposure and between 5.1 and 9.3 for neighbourhood exposure. Asbestos refers to a group of thin fibres: chrysotile, crocidolite, amosite, tremolite, anthophylite and actinolite. Nearly $95 \%$ of the asbestos used worldwide is chrysotile and most others fibres are commonly used with chrysotile. Other risk factors have been incriminated [8]. A high level of evidence is associated with environmental exposure to erionite or fluoroedenite and for irradiation [9] while controversial data supported the role of man-made ceramic fibres or viral contamination by simian virus 40 (SV40). Further genetic predisposition is suggested for increased susceptibility to mineral-fibre carcinogenesis [10].

\section{Symptoms}

Mesothelioma affects mainly older men [4] aged 60-70 years [5]. The prominent symptoms that could last from months to 1-2 yrs before diagnosis are chest pain and shortness of breath, affecting more than half the patients, while constitutional symptoms (fever, weight loss and fatigue) are less commonly observed [11]. At the difference of lung cancer, cough is rarely a cardinal sign at initial presentation. Characteristically, MPM has usually a local spread, growing along the pleural surface, extending to the opposite pleura or to the thoracic wall with possible involvement of the pericardium and of the diaphragm. Regional lymph node infiltration is common (34-50\% of patients). Distant metastases are rare events [11]. Local tumour spread can result in oesophageal compression and dysphagia, medullar compression or plexopathy, vena cava syndrome, cardiac arrhythmia or pericardial tamponade.

\section{Diagnostic procedures and histological diagnosis}

Diagnosis begins with a chest radiograph which typically demonstrates pleural thickening or pleural effusion [12]. Unfortunately, it is not suitable for an early diagnosis. Computed tomography (CT) is more sensitive and specific in assessing pleural diseases. Common CT findings in patients with MPM are thickening of the pleural surfaces, pleural calcifications and pleural effusions [13]. Contraction of the hemithorax is often noted and contralateral mediastinal shift occurs in a little number of cases. Disease beyond the parietal pleura is found in the chest wall, mediastinum, lymph nodes and diaphragm. Limitations of the CT in staging MPM concerned the assessment of chest wall invasion, the detection of pathological mediastinal lymph nodes, the evaluation of diaphragmatic extension, and the detection of peritoneal studding and solid organ metastases $<2 \mathrm{~mm}$ in size. To improve the accuracy of $\mathrm{CT}_{\text {, some authors proposed the }}$ use of magnetic resonance imaging (MRI) in addition to or in place of CT. MRI has not been demonstrated to be better than $\mathrm{CT}$ in staging MPM, essentially in determining nodal involvement. Nevertheless, MRI is superior to CT in revealing solitary foci of chest wall invasion, endothoracic fascia involvement and diaphragmatic muscle invasion [14]. For all these reasons, $\mathrm{CT}$ remains the standard imaging diagnostic procedure with MRI allowing additional information to be obtained when surgery is considered (assessment of chest wall and diaphragmatic invasion). Promising data came from the use of ${ }^{18} \mathrm{~F}$-fluorodeoxyglucose positron emission tomography (PET scan) for defining mediastinal lymph node involvement $[15,16]$ and finding extrathoracic occult extension $[16,17]$.

In their recommendations, the European Respiratory Society (ERS) and the European Society of Thoracic Surgery (ESTS) suggested a three-step staging, pretherapeutic investigations depending on the choice of treatment with radical or palliative intent [18]. Step I will be considered for all patients (demographics, clinical history and physical examination, chest radiograph and blood tests); step II (at least chest and upper abdomen CTscan and pulmonary function tests) for those who will receive any kind of treatment; and step III for the minority of patients considered for radical and/or combined modality therapy, including invasive tests assessing mediastinum (endobronchial ultrasound (EBUS), mediastinoscopy), extrathoracic (FDG-PET) or diaphragmatic (MRI) spread. There are at least five different staging systems available in MPM. The recommended one, developed by the International Mesothelioma Interest Group, is approved by the Union Internationale Contre le Cancer 
(UICC) [19]. However, the inaccuracy in assessing the $\mathrm{T}$ and $\mathrm{N}$ descriptors underlines the need for the development of a robust new staging system, prospectively validated applicable as well for clinicians and surgeons.

The ERS/ESTS Task Force also recommended using CT for response assessment [18]. However, there was no definite consensus on the criteria that should be used for radiological measure ment. Indeed, the growth pattern of MPM is not well suited by the World Health Organization (WHO) criteria, which need bidimensional measurements, or by the more recent response evaluation criteria in solid tumours (RECIST) or modified RECIST criteria which only require a unidimensional measurement. The interpretation of these criteria in MPM may be difficult: which lesions should be considered as targets and what constitutes the longest diameter of the lesion? While potentially interesting, the contribution of PET or combined PET-CT to response assessment remains under investigation.

The histological diagnosis of MPM is of particular importance but is often difficult. Differential diagnosis has to be made with benign inflammatory and reactive lesions of the pleura (pleural effusion during cardiac failure, autoimmune diseases, etc.) and pleural metastases that the primary tumour is most frequently the lung or the breast, although others could present with a pseudomesotheliomatous aspect (thymomas, angiosarcomas, etc.). The International Mesothelioma Panel identified recommendations [20] that were applied in the guidelines developed by the ERS/ESTS Task Force [18]. To consider the diagnosis of mesothelioma, it is recommended to take deep and large biopsies including fat and/or muscle, to assess the degree of invasiveness, of both macroscopically normal and abnormal appearing pleura. The WHO classification had to be used, distinguishing the three main histological subtypes of mesothelioma: epitheloid, which accounts for more than $60 \%$ of the cases, sarcomatoid and mixed or biphasic histology. The guidelines suggested that two markers with positive and two others with negative predictive value were used, differentiating epitheloid MPM from adenocarcinoma and for sarcomatoid MPM, to use two broad-spectrum anti-cytokeratin antibodies and two markers with negative predictive value.

As the diagnosis of MPM is still a challenge, authors searched for pleural or serum markers differentiating between benign effusions, pleural metastases and MPM. Mainly, two proteins have been investigated, osteopontin, the interest of which is now decreased and mesothelin. In some series, soluble mesothelin related protein in fluids (pleural or peritoneal) and serum showed a good specificity with sub-optimal sensitivity [21-23]. There is some evidence that pleural fluid mesothelin can help in distinguishing mesothelioma from metastatic carcinomas and benign effusions, with intraindividual reproducibility, remaining reliable after pleurodesis [24]. There are also arguments for an association between serum mesothelin and disease progression [25].

\section{Prognosis}

Although the prognosis of MPM is globally poor with a median survival time (MST) of around 12 months, there remain large discrepancies among patients. Various clinical prognostic factors [26] have been considered such as performance status, stage and weight loss. However, histology consistently is associated with prognosis, nonepitheloid types presenting with poorer survival. Other prognostic factors were not uniformly observed throughout all series as age, sex, symptoms and quality-oflife parameters. Among biological factors, haemoglobin level, lactose dehydrogenase $(\mathrm{LDH})$, white blood cell and thrombocyte counts were regularly related to prognosis. The prognostic role of serum biomarkers as soluble mesothelin and osteopontin are under investigation. Among all these factors, histology and disease extent appears the most useful for determining the therapeutic strategy. For example, SUGARBAKER et al. [27] showed in a patient population who underwent extrapleural pneumonectomy, chemotherapy, and radiotherapy that the poorest survival rate was in patients with nonepithelial MPM variant (OR for death 3.0, 95\% Cl 2.0-4.5; $\mathrm{p}<0.0001)$, positive resection margins $(\mathrm{OR} \quad 1.7,95 \% \mathrm{Cl}$ 1.2-2.6; $\mathrm{p}=0.008)$ and extrapleural metastatic nodes (OR 2.0, 95\% Cl 1.3-3.2; $p=0.003$ ). This observation was further confirmed in a systematic review including series assessing prognostic factors for patients who underwent extrapleural pneumonectomy [28]. Patients with non-epithelial histotype and nodal involvement consistently demonstrated the poorest outcome. Two main prognostic scoring systems have been proposed by the Cancer and Leukemia Group B (CALGB) [29] and the European Organisation for Research and Treatment of Cancer (EORTC) [30] that was further adapted [31]. The CALGB was able on the basis of simple clinical (performance status, age, thoracic pain, weight loss) and biological (haemoglobinaemia, white cell count) factors to separate 


\section{Educational questions}

1) A 58-yrold man consulted for shortness of breath. He has been exposed to asbestos 30 yrs ago. Right pulmonary sounds were reduced and a large pleural effusion and pleural calcifications were noted on chest radiograph. Which of the following affirmation is correct?

a) The diagnostic of mesothelioma can be definitively claimed without any further investigations.

b) The diagnostic of mesothelioma can be assessed on a cytological examination of the pleural fluid. There is no need for immunohistochemistry testing.

c) Chest $C T$ is useful only if surgery is considered.

d) Large and multiple biopsies obtained during a thoracoscopic procedure are the bestway to obtain a definite mesothelioma diagnosis.

2) Among the prognostic factors that have been identified in malignant pleural mesothelioma, which one has both prognostic and operational value?
a) Histology
b) Age
c) Sex
d) Performance status
e) Propositions 1 and 4 are both correct

the patients into six groups with distinct prognosis, MST ranging from 13.9 months in the best prognostic group to 1.4 months in the poorest one. Another prognostic index was developed by the EORTC, based on three major independent prognostic factors (performance status, histology and stage), considering progression-free survival as the main end-point [32].

\section{Treatment}

For the majority of solid tumours, three therapeutic options are commonly available for those with MPM: surgery, radiotherapy and chemotherapy, including "biological" and "targeted" therapies. As will be further discussed, the evidences supporting the activity of these treatments in MPM are limited. Due to the paucity of data, the ERS/ESTS Task Force [18] recommended that at least all patients received best supportive care and that any kind of treatment was decided on a case-by-case basis taking into account the risks and benefits of each procedure after discussing with the patients and/or their relatives. It was also recommended to consider the performance status and the patient's comorbidities that could impact on the performances of the subsequent therapy.

\section{Surgery}

Three surgical procedures can be offered to the MPM patients: pleurectomy, pleurectomy-decortication and extrapleural pneumonectomy (EPP). The first two mainly have a palliative role in controlling pleural effusion in case of failure of chemical pleurodesis. EPP consists in an en bloc pleuro-pulmonary resection, extended to the pericardium and the diaphragm. The curative role of EPP was first suggested in a small series of highly selected patients. A systematic review of EPP was recently published [33]. From 58 series, the authors observed a MST ranging from 9.4 to 27.5 months and a $5-y r$ survival of $24 \%$. There is clearly a need to better define the patients for whom EPP can be proposed (epithelial subtype, good performance status, adequate lung function, absence of nodal mediastinal involvement, etc.). The effectiveness of EPP must also be counterbalanced with its sideeffects and a relative high mortality rate, estimated in the expert surgical teams between 5 and $10 \%$, which can be reduced by a training effect. For example, perioperative mortality reported by the Sugarbaker's team was 6\% in 1991 and progressively diminished to $3.4 \%$ in their last publication [27]. Nevertheless, postoperative complications are frequent, with more than half the patients presenting with cardiac arrhythmias, pulmonary embolism or bronchopleural fistula. The efficacy and the impact on overall survival of EPP need confirmation in a prospective randomised study. This study (ClinicalTrials.gov identifier NCT00253409) is currently conducted by the UK Institute of Cancer Research. After three cycles of a platinum-based chemotherapy, cisplatin or carboplatin, patients with resectable MPM (T1-3, NO-1, MO) were randomised between EPP followed by postoperative radiotherapy or follow-up only. The authors planned to recruit 670 patients. The trial started in 2005 and is now closed to recruitment. The results are forthcoming.

Due to disappointing results of surgery alone, multimodality treatment combining surgery, chemotherapy and postoperative radiotherapy was tested, essentially in small size retrospective series [34]. Although large heterogeneity in therapeutic modalities, disease stage and histological repartition, they did not clearly show a survival improvement in comparison with surgery alone whatever the combination used: surgery plus postoperative radiotherapy; surgery plus chemotherapy (intravenous or intrapleural); or with a trimodality approach. The administration of a photosensitiser in addition to surgery increased lifethreatening sideeffects without improving overall survival. The ERS/ESTS Task Force recommended that patients, candidates for combined modality treatment, are included in clinical trials [18].

\section{Radiotherapy}

Overall, the usefulness of chest radiotherapy in MPM has not been demonstrated. Currently, it is not recommended to apply radiotherapy with "curative" intent to the tumour in situ because of the technical constraints, needing the whole rib cage to be irradiated while MPM has only a weak radio-sensitivity [35]. Data from the literature concerning postoperative radiotherapy and intensity modulated radiation therapy (IMRT) are scarce, not allowing their use outside of clinical studies [35]. The Swiss Group for Clinical Cancer Research assess in an ongoing phase II randomised study (ClinicalTrials.gov identifier NCT00334594) the role of postoperative radiotherapy after induction chemotherapy and macroscopically complete resection of the tumour. A palliative role of radiotherapy can be 
considered for painful parietal infiltration or permeation nodules. The efficacy of radiotherapy in preventing permeation nodules remains debatable. A French randomised study first showed that prophylactic irradiation ( $3 \times 7$ Gy) could dramatically reduce malignant seeding along invasive thoracic procedures from 40\% (observation arm) to $0 \%$ (radiotherapy arm) $(p<0.001)$ [36]. A second small-sized randomised study did not demonstrate any effect of a single dose of 10 Gy [37]. A further randomised study including 62 patients [38], using a similar radiotherapy schedule $(3 \times 7 \mathrm{~Gy})$ as in the first study, could not find a significant difference in the risk of tract metastasis associated with drain site between the two arms $(p=0.75)$.

\section{Chemotherapy}

For a long time, MPM has been considered a refractory disease to any kind of treatment, in particular to chemotherapy. Compared with lung cancer, for which more than 15 randomised trials have demonstrated an improvement in overall survival for patients receiving chemotherapy compared with best supportive care, there are only limited data in MPM. Currently, only one British randomised trial has been performed [39]. Patients received either 12 weekly cycles of vinorelbine monotherapy, four cycles of combined mitomycin, vinblastine and cisplatin (MVP) or symptomatic care only. No statistically significant improvement in overall survival was observed when combining both chemotherapy arms (MST 8.5 months) compared with the BSC arm (MST 7.6 months) $(p=0.32)$. There was also no significant improvement of symptom control except for chest pain and sweating which were reduced with the cisplatin regimen. It must be highlighted that the trial was prematurely stopped due to lack of accrual and that the choice of the chemotherapy regimen was perhaps not optimal. In a systematic literature review of phase II studies, the evidencebased group of the European Lung Cancer Working Party showed that cisplatin-based regimens offered the highest response rate (RR) in unresectable MPM. The best combinations showing RR around 30\% were cisplatin plus either doxorubicin, etoposide or gemcitabine [40]. A further argument favouring the use of chemotherapy in MPM patients came from a small randomised study [41]. After symptom control, patients were randomised between immediate or delayed chemotherapy (MVP) at the time of symptomatic progression. 43 patients were eligible. In the delayed arm, $77 \%$ of the patients received chemotherapy, and $100 \%$ in the immediate arm. Time to symptomatic progression was increased ( 25 versus 11 weeks) and survival was improved (MST 14 versus 10 months; 1-yr sunvival 66 versus 36\%), without reaching statistical significance $(p=0.1)$ probably because the study was not powered for. Two randomised phase III trials were published in the 2000s $[42,43]$. These two studies compared a doublet regimen to cisplatin alone. As suggested by our meta-analysis [40], cisplatin monotherapy cannot be considered an effective treatment in MPM. Indirectly, these two trials supported the use of cisplatin-based $\left(75-80 \mathrm{mg} \cdot \mathrm{m}^{-2}\right)$ doublet chemotherapy in adequate performance status patients with MPM, if we extrapolate thatcisplatin alone is similar to a no-treatment decision.

\section{When the decision to administer chemotherapy is made, what is the best regimen?}

In the first phase III trial [42], 448 patients were randomised between cisplatin or cisplatin plus pemetrexed. All end-points were significantly better with the doublet: RR (41.3 versus 16.7\%; $p<0.001$ ), sunvival (MST 12.1 versus 9.3 months; 1 -yr survival 50 versus $38 \% ; p=0.02$ ) and timetoprogression (median 5.7 versus 3.9 months; $p=0.001$ ) despite a significant increase in haematological and digestive toxicities. Pemetrexed toxicity being reduced with concomitant administration of folate and vitamin $\mathrm{B} 12$, the authors were able to show that the effectiveness of the combination was not reduced in the subgroup of patients treated with this supplementation. In the second European trial [43], 250 patients received cisplatin alone or cisplatin plus raltitrexed. The same conclusions on the efficacy of the doublet can be drawn with significant improvement in RR ( 23.6 versus $13.6 \% ; p=0.06$ ), survival (MST 11.4 versus 8.8 months, 1-yr survival 46 versus $40 \%$; $p=0.05$ ) and disease-free survival (median 5.3 versus 4 months; $p=0.06$ ). Although the currently accepted standard chemotherapy regimen is cisplatin plus pemetrexed (with folate and vitamin B12 supplementation), other chemotherapy schedules, have shown some activity in phase II studies and could be considered for further testing: cisplatin-gemcitabine, cisplatin-etoposide, cisplatin-doxorubicin, cisplatin-interferon, oxaliplatin plus raltitrexed (or gemcitabine or vinorelbine) and methotrexate [40].

Despite these encouraging improvements during the last decade, overall results remain in some way disappointing and further studies are
3) A 55-yrold man in good performance status (90 on the Karnofsky scale) presented with a stage II epitheloid mesothelioma. ${ }^{18}$ F-FDGPET-CT and cenvical mediastinoscopy did not show any neoplastic nodal involvement. Lung and heart functions were normal. Based on the ERS/ESTS guidelines, which will be the more appropriate therapeutic option?

a) Pleurectomy-decortication

b) Pleurectomy followed by cisplatin-based chemotherapy

c) Inclusion in a clinical trial testing a combined modality approach with extrapleural pneumonectomy

d) Treatment by IMRT

e) None of the therapeutic options can be proposed

4) A 65-yrold man presented with stage IV unresectable mesothelioma. There is no functional contraindication for chemotherapy administration. Which is the best option?

a) Best supportive care only

b) Cisplatin plus pemetrexed whatever the histologic subtype of mesothelioma, all being equally sensitive to chemotherapy

c) Cisplatin plus pemetrexed in case of epitheloid mesothelioma

d) Carboplatin plus pemetrexed in case of epithe loid mesothelioma 
needed. A first question is the substitution of cisplatin, which has well known chronic digestive, neurologic and renal toxicities, by carboplatin. Some data suggested the usefulness of carboplatin with pemetrexed [44]. In an expanded access programme, patients received pemetrexed with either cisplatin or carboplatin [45]. While this study was not randomised, RR and survival appeared similar whatever the type of platinum derivative. As there is no formal comparison between these two drugs, the use of carboplatin cannot be recommended and should be discussed on a case-by-case basis. Other approaches that have been tested, included immunomodulatory agents, mainly interferons and interleukins. Some activity has been reported but it was not possible to draw definite conclusions on these drugs because of the heterogeneity in their administration routes (pleural, sub-cutaneous, intramuscular, intravenous), the type of products, the eventual combination with other drugs and the case-mix, etc., the disease stage or the repartition of histological subtypes that largely differ from one study to another [40]. Other immunomodulatory methods are currently under investigation as autologous dendritic cells administration (www.clinicaltrials.gov).

New approaches based on a better knowledge of the molecular pathways involved in the MPM carcinogenic process will help in finding more active therapeutics [46]. As for breast or lung cancers, some targeted therapies have been tested unfortunately without any significant efficacy: gefitinib and erlotinib despite the expression of EGFR on MPM cells, and imatinib or thalidomide, an antiogenic drug. Ongoing studies are testing other molecules, such as bortezomib, a proteasome inhibitor, everolimus, a mTOR inhibitor, dasatinib which acts on the BCR/ABL kinase activity or tyrosine kinase inhibitors, such as vandetanib, sunitinib or sorafenib. Bevacizumab, an anti-vascular endothelial growth factor (VEGF) monoclonal antibody, in addition to cisplatinbased chemotherapy, showed no significant activity ( $R R$, survival) in a phase II randomised trial. Nevertheless, a French randomised study (MAPS; ClinicalTrials.gov identifier NCT00651456) comparing cisplatin-pemetrexed with or without bevacizumab is underway. The phase II has been completed and the phase III is ongoing.

Epigenetic regulation of tumoursuppressor genes through chromatin condensation and decondensation has emerged as an important mechanism that leads to tumourigenesis. Epigenetic modulation is currently under investigation. For example, histone deacetylases (HDAC) are major regulators of gene expression that can affect the cell functioning as well as differentiation and proliferation. A gene-activation therapy by HDAC inhibition (HDACi) could induce cell modifications and show potential anticancer activity. HDACi includes a variety of compounds belonging to several structural classes: hydroxamic acids (TSA or trichostatin A, suberoylanilide hydroxamic acid (SAHA) or vorinostat); cyclic peptides (trapoxin, depsipeptide), benzamides (MS-27-275) and short-chain fatty acids (butyric acid and valproic acid). HDACi demonstrated in vitro and in clinical trials some potential anticancer activity in haematological and solid tumours [47]. Vorinostat (SAHA) monotherapy has been tested so far in patients with MPM failing after standard firstline chemotherapy. In a phase I trial [48], two patients out of 13 demonstrated a partial response. The results of a phase II trial (oral vorinostat versus placebo) are not published and a placebo-controlled randomised phase III study of second-line oral vorinostat is ongoing. Another HDACi monotherapy with belinostat (PXD101) showed no antitumour activity in MPM, with two patients among 13 presenting with a stable disease [49]. In a phase II study including refractory or progressing heavily pre-treated MPM, the combination of valproate and doxorubicin showed a promising 16\% RR activity in good performance status patients [50].

\section{Salvage chemotherapy in MPM?}

Currently, there is no standard second-line therapy for MPM [18]. In a phase III trial comparing pemetrexed to palliative care alone, JASSEM et al. [51] demonstrated improved progression-free survival and time to progression without any significant impact on overall survival. In the few other studies assessing the effectiveness of secondline chemotherapy, RR between 10 and 20\% were noted with doxorubicin, pemetrexed, pemetrexed plus carboplatin, vinorelbine or a combination of cisplatin, mitomycin and irinotecan.

\section{Conclusions}

MPM is an important public health problem, not only in industrialised but also in emerging countries. Although progress has been made during the last decade, MPM is still a difficulttodiagnose and difficultto-treat cancer. Outside of politic decisions banning the use of asbestos, the principal aetiological agent of MPM, research is imperatively needed to improve the prognosis and the quality of life of the patients presenting with this debilitating disease. 


\section{References}

1. Walker AM, Loughlin JE, Friedlander ER, et al. Projections of asbestos-related disease 1980-2009. J Occup Med 1983; 25: 409-425.

2. Wagner JC, Sleggs CA, Marchand P. Diffuse pleural mesothelioma and asbestos exposure in the North Western Cape Province. Br J Ind Med 1960; 17: 260-271.

3. Lanphear BP, Buncher CR. Latent period for malignant mesothelioma of occupational origin. J Occup Med 1992; 34: 718-721.

4. Hodgson JT, McElvenny DM, Darnton AJ, et al. The expected burden of mesothelioma mortality in Great Britain from 2002 to 2050. Br J Cancer 2005; 92: 587-593.

5. Tan E, Warren N, Darnton AJ, et al. Projection of mesothelioma mortality in Britain using Bayesian methods. $\mathrm{Br} \mathrm{J}$ Cancer 2010; 103: 430-436.

6. Montanaro F, Bray F, Gennaro V, et al. Pleural mesothelioma incidence in Europe: evidence of some deceleration in the increasing trends. Cancer Causes Control 2003; 14: 791-803.

7. Price B, Ware A. Mesothelioma trends in the United States: an update based on Surveillance, Epidemiology, and End Results Program data for 1973 through 2003. Am J Epidemiol 2004; 159: 107-112.

8. Weiner SJ, Neragi-Miandoab S. Pathogenesis of malignant pleural mesothelioma and the role of environmental and genetic factors. J Cancer Res Clin Oncol 2009; 135: 15-27.

9. Carbone M, Kratzke RA, Testa JR. The pathogenesis of mesothelioma. Semin Oncol 2002; 29: 2-17.

10. Carbone M, Emri S, Dogan AU, et al. A mesothelioma epidemic in Cappadocia: scientific developments and unexpected social outcomes. Nat Rev Cancer 2007; 7: 147-154.

11. Pass HI, Vogelzang N, Hahn S, et al. Malignant pleural mesothelioma. Curr Probl Cancer 2004; 28: 93-174.

12. Wechsler RJ, Rao VM, Steiner RM. The radiology of thoracic malignant mesothelioma. Crit Rev Diagn Imaging 1984; 20: $283-310$.

13. Gamsu G, Aberle DR, Lynch D. Computed tomography in the diagnosis of asbestos-related thoracic disease. J Thorac Imaging 1989; 4: 61-67.

14. Patz EF Jr, Shaffer K, Piwnica-Worms DR, et al. Malignant pleural mesothelioma: value of CT and MR imaging in predicting resectability. Am J Roentgenol 1992; 159: 961-966.

15. Benard F, Sterman D, Smith RJ, et al. Metabolic imaging of malignant pleural mesothelioma with fluorodeoxyglucose positron emission tomography. Chest 1998; 114: 713-722.

16. Subramaniam RM, Wilcox B, Aubry MC, et al. ${ }^{18} \mathrm{~F}$-fluoro-2-deoxy-D-glucose positron emission tomography and positron emission tomography/computed tomography imaging of malignant pleural mesothelioma. J Med Imaging Radiat Oncol 2009; 53: 160-169.

17. Schneider DB, Clary-Macy C, Challa S, et al. Positron emission tomography with ${ }^{18} \mathrm{~F}$-fluorodeoxyglucose in the staging and preoperative evaluation of malignant pleural mesothelioma. J Thorac Cardiovasc Surg 2000; 120: 128-133.

18. Scherpereel A, Astoul P, Baas P, et al. Guidelines of the European Respiratory Society and the European Society of Thoracic Surgeons for the management of malignant pleural mesothelioma. Eur Respir J 2010; 35: 479-495.

19. Wittekind C, Greene F, Hutter R, et al. TNM Atlas. In: UICC, International Union Against Cancer, editors. Illustrated Guide to the TMN Classification of Malignant Tumours. 5th edn. Berlin, Springer, 2004. pp 169-176.

20. Husain AN, Colby TV, Ordonez NG, et al. Guidelines for pathologic diagnosis of malignant mesothelioma: a consensus statement from the International Mesothelioma Interest Group. Arch Pathol Lab Med 2009; 133: 1317-1331.

21. Robinson BW, Creaney J, Lake R, et al. Mesothelin-family proteins and diagnosis of mesothelioma. Lancet 2003; 362: 1612-1616.

22. Scherpereel A, Grigoriu B, Conti M, et al. Soluble mesothelin-related peptides in the diagnosis of malignant pleural mesothelioma. Am J Respir Crit Care Med 2006; 173: 1155-1160.

23. Wheatley-Price P, Yang B, Patsios D, et al. Soluble mesothelin-related Peptide and osteopontin as markers of response in malignant mesothelioma. J Clin Oncol 2010; 28: 3316-3322.

24. Davies HE, Sadler RS, Bielsa S, et al. Clinical impact and reliability of pleural fluid mesothelin in undiagnosed pleural effusions. Am J Respir Crit Care Med 2009; 180: 437-444.

25. Grigoriu BD, Chahine B, Vachani A, et al. Kinetics of soluble mesothelin in patients with malignant pleural mesothelioma during treatment. Am J Respir Crit Care Med 2009; 179: 950-954.

26. Steele JP. Prognostic factors for mesothelioma. Hematol Oncol Clin North Am 2005; 19: 1041-1052vi.

27. Sugarbaker DJ, Flores RM, Jaklitsch MT, et al. Resection margins, extrapleural nodal status, and cell type determine postoperative long-term survival in trimodality therapy of malignant pleural mesothelioma: results in 183 patients. J Thorac Cardiovasc Surg 1999; 117: 54-63.

28. Cao C, Yan TD, Bannon PG, et al. Summary of prognostic factors and patient selection for extrapleural pneumonectomy in the treatment of malignant pleural mesothelioma. Ann Surg Oncol 2011; Epub ahead of print, DOI: 10.1245/s10434-011-1728-x.

29. Herndon JE, Green MR, Chahinian AP, et al. Factors predictive of survival among 337 patients with mesothelioma treated between 1984 and 1994 by the Cancer and Leukemia Group B. Chest 1998; 113: 723-731.

30. Curran D, Sahmoud T, Therasse P, et al. Prognostic factors in patients with pleural mesothelioma: the European Organization for Research and Treatment of Cancer experience. J Clin Oncol 1998; 16: 145-152.

31. Bottomley A, Coens C, Efficace F, et al. Symptoms and patient-reported well-being: do they predict survival in malignant pleural mesothelioma? A prognostic factor analysis of EORTC-NCIC 08983: randomized phase III study of cisplatin with or without raltitrexed in patients with malignant pleural mesothelioma. J Clin Oncol 2007; 25: 5770-5776.

32. Francart J, Vaes $E$, Henrard $S$, et al. A prognostic index for progression-free survival in malignant mesothelioma with application to the design of phase II trials: A combined analysis of 10 EORTC trials. Eur J Cancer 2009; 45: 2304-2311.

33. Cao CQ, Yan TD, Bannon PG, et al. A systematic review of extrapleural pneumonectomy for malignant pleural mesothelioma. J Thorac Oncol 2010; 5: 1692-1703.

34. Sugarbaker DJ, Wolf AS. Surgery for malignant pleural mesothelioma. Expert Rev Respir Med 2010; 4: 363-372.

35. Scherpereel A, [The experts' conference of the Societe de Pneumologie de Langue Francaise (SPLF) on malignant pleural mesothelioma (MPM): useful and necessary recommendations.] Rev Mal Respir 2006; 23: 11S5-11S6.

36. Boutin C, Rey F, Viallat JR. Prevention of malignant seeding after invasive diagnostic procedures in patients with pleural mesothelioma. A randomized trial of local radiotherapy. Chest 1995; 108: 754-758. 
37. Bydder S, Phillips M, Joseph DJ, et al. A randomised trial of single-dose radiotherapy to prevent procedure tract metastasis by malignant mesothelioma. Br J Cancer 2004; 91: 9-10.

38. O'Rourke N, Garcia JC, Paul J, et al. A randomised controlled trial of intervention site radiotherapy in malignant pleural mesothelioma. Radiother Oncol 2007; 84: 18-22.

39. Muers MF, Stephens RJ, Fisher P, et al. Active symptom control with or without chemotherapy in the treatment of patients with malignant pleural mesothelioma (MS01): a multicentre randomised trial. Lancet 2008; 371: 1685-1694.

40. Berghmans T, Paesmans M, Lalami Y, et al. Activity of chemotherapy and immunotherapy on malignant mesothelioma: a systematic review of the literature with meta-analysis. Lung Cancer 2002; 38: 111-121.

41. $O^{\prime}$ Brien $M E$, Watkins $D$, Ryan $C$, et al. A randomised trial in malignant mesothelioma $(M)$ of early $(E)$ versus delayed (D) chemotherapy in symptomatically stable patients: the MED trial. Ann Oncol 2006; 17: 270-275.

42. Vogelzang NJ, Rusthoven JJ, Symanowski J, et al. Phase III study of pemetrexed in combination with cisplatin versus cisplatin alone in patients with malignant pleural mesothelioma. J Clin Oncol 2003; 21: 2636-2644.

43. van Meerbeeck JP, Gaafar R, Manegold C, et al. Randomized phase III study of cisplatin with or without raltitrexed in patients with malignant pleural mesothelioma: an intergroup study of the European Organisation for Research and Treatment of Cancer Lung Cancer Group and the National Cancer Institute of Canada. J Clin Oncol 2005; 23: $6881-6889$.

44. Castagneto B, Botta M, Aitini E, et al. Phase II study of pemetrexed in combination with carboplatin in patients with malignant pleural mesothelioma (MPM). Ann Oncol 2008; 19: 370-373.

45. Santoro A, O'Brien ME, Stahel RA, et al. Pemetrexed plus cisplatin or pemetrexed plus carboplatin for chemonaive patients with malignant pleural mesothelioma: results of the International Expanded Access Program. $J$ Thorac Oncol 2008; 3: 756-763.

46. Zucali PA, Ceresoli GL, De Vincenzo F, et al. Advances in the biology of malignant pleural mesothelioma. Cancer Treat Rev 2011; Epub ahead of print, PMID, 21288646.

47. Stimson L, Wood V, Khan 0, et al. HDAC inhibitor-based therapies and haematological malignancy. Ann Oncol 2009; 20: $1293-1302$.

48. Krug LM, Curley T, Schwartz L, et al. Potential role of histone deacetylase inhibitors in mesothelioma: clinical experience with suberoylanilide hydroxamic acid. Clin Lung Cancer 2006; 7: 257-261.

49. Ramalingam SS, Belani CP, Ruel C, et al. Phase II study of belinostat (PXD101), a histone deacetylase inhibitor, for second line therapy of advanced malignant pleural mesothelioma. J Thorac Oncol 2009; 4: 97-101.

50. Scherpereel A, Berghmans T, Lafitte JJ, et al. Valproate-doxorubicin: promising therapy for progressing mesothelioma. A phase II study. Eur Respir J 2011; 37: 129-135.

51. Jassem J, Ramlau R, Santoro A, et al. Phase III trial of pemetrexed plus best supportive care compared with best supportive care in previously treated patients with advanced malignant pleural mesothelioma. $\mathrm{J}$ Clin Oncol 2008; 26: $1698-1704$. 